\title{
A!
}

This is an electronic reprint of the original article.

This reprint may differ from the original in pagination and typographic detail.

Koponen, Laura; Tunturivuori, Lasse O.; Puska, Martti J.; Nieminen, Risto M.

\section{Effect of the surrounding oxide on the photoabsorption spectra of Si nanocrystals}

Published in:

Physical Review B

DOI:

10.1103/PhysRevB.79.235332

Published: 26/06/2009

Document Version

Publisher's PDF, also known as Version of record

Please cite the original version:

Koponen, L., Tunturivuori, L. O., Puska, M. J., \& Nieminen, R. M. (2009). Effect of the surrounding oxide on the photoabsorption spectra of Si nanocrystals. Physical Review B, 79(23), 1-6. [235332].

https://doi.org/10.1103/PhysRevB.79.235332

This material is protected by copyright and other intellectual property rights, and duplication or sale of all or part of any of the repository collections is not permitted, except that material may be duplicated by you for your research use or educational purposes in electronic or print form. You must obtain permission for any other use. Electronic or print copies may not be offered, whether for sale or otherwise to anyone who is not an authorised user. 


\title{
Effect of the surrounding oxide on the photoabsorption spectra of Si nanocrystals
}

\author{
Laura Koponen, * Lasse O. Tunturivuori, Martti J. Puska, and Risto M. Nieminen \\ Department of Applied Physics, Helsinki University of Technology, P.O. Box 1100, 02015 TKK, Finland \\ (Received 5 March 2009; revised manuscript received 29 May 2009; published 26 June 2009)
}

\begin{abstract}
A systematic study of the optical absorption of small silicon nanocrystals ( $\mathrm{Si}-\mathrm{NCs}$ ) embedded in silicon dioxide is performed using real-time time-dependent density-functional theory. The modeled Si-NCs contain up to $47 \mathrm{Si}$ atoms with the surrounding oxide being described by a shell of $\mathrm{SiO}_{2}$. The oxide-embedded $\mathrm{Si}-\mathrm{NCs}$ exhibit absorption spectra that differ significantly from the spectra of the hydrogen-passivated Si-NCs. In particular, the minimum absorption energy is found to decrease when the Si-NCs are exposed to dioxide coating. Unexpectedly, the absorption energy of the oxide-embedded Si-NCs remains approximately constant for core sizes down to 17 atoms, whereas the absorption energy of the hydrogen-passivated Si-NCs increases with decreasing crystal size. This trend suggests a different mechanism for producing the lowest-energy excitations in these two cases.
\end{abstract}

DOI: 10.1103/PhysRevB.79.235332

PACS number(s): 78.67.Bf, 73.20.At, 73.22.-f, 31.15.ee

\section{INTRODUCTION}

Silicon-based photonics is an emerging field of nanotechnology with extensive experimental and theoretical research activity. ${ }^{1}$ In low-dimensional silicon structures, electronic and optical properties can be tuned as a function of size and, in addition, new phenomena appear. Nowadays there exist several methods to fabricate silicon nanocrystals ( $\mathrm{Si}-\mathrm{NCs}$ ), both in the porous form ${ }^{2,3}$ and as embedded in amorphous silicon dioxide. ${ }^{4-7}$ The size of the Si-NCs can be controlled down to nanometer or even subnanometer accuracy.

The indirect energy gap of crystalline silicon makes it a poor light emitter. In 1990 it was observed experimentally by Canham, ${ }^{2}$ however, that in porous silicon, photoluminescence occurs in the visible range at room temperature. Later, Wolkin et $a .^{3}{ }^{3}$ demonstrated that exposure of the clean porous-silicon surface to oxygen redshifts the photoluminescence by as much as $1 \mathrm{eV}$.

Free-standing Si-NCs show strong luminescence, the color of which depends on the size of the NCs. Such systems have been extensively studied computationally and obey the trend of increasing gap and absorption energies as their size is reduced, suggesting that quantum confinement is responsible for the optical activity. ${ }^{8,9}$ The effect of surface reconstruction is addressed in Ref. 10, where a considerable redshift of the optical gap is observed compared to unreconstructed Si-NCs. Moreover, several computational studies have been conducted concerning oxygen-associated centers at the $\mathrm{Si}-\mathrm{NC}$ surface, e.g., for $\mathrm{Si}-\mathrm{O}-\mathrm{Si}$ defects and for single and multiple $\mathrm{Si}=\mathrm{O}$ defects. ${ }^{11-16}$ Significant changes in the electronic properties including absorption spectra are recorded, especially in the case of double-bonded oxygen defects.

A less studied but more interesting case in view of suggested applications are the Si-NCs embedded in silicon dioxide. Light amplification in oxide-embedded $\mathrm{Si}-\mathrm{NCs}$ was demonstrated by Pavesi et al. ${ }^{17}$ thus providing a starting point for the development of the silicon laser. Localized interface states were suggested to explain the optical behavior of this system. On the other hand, the interplay between quantum confinement and defect-related effects has been demonstrated experimentally by Godefroo et al. ${ }^{18}$ and has also been used to explain these properties. In their work, oblate Si-NCs with a diameter of about $3 \mathrm{~nm}$ embedded in silicon oxide were studied and the origin of photoluminescence was traced back to defects with dangling bonds. As these systems are passivated with hydrogen, threedimensional quantum confinement becomes responsible. Daldosso et al. ${ }^{19}$ have investigated by static densityfunctional theory (DFT) methods the $\mathrm{Si}_{10}-\mathrm{NC}$ in an ideal $\mathrm{SiO}_{2}$ lattice. Their calculation shows a redshift of $2 \mathrm{eV}$ in the energy gap between the highest occupied molecular orbital (HOMO) and lowest unoccupied molecular orbital (LUMO) orbitals and thus in the minimum absorption energy compared to the hydrogen-passivated Si-NC. Ramos et al..$^{20,21}$ mimic in their computational study the effect of the silicon dioxide matrix by adding one or two oxide shells around the $\mathrm{Si}_{5^{-}}, \mathrm{Si}_{17^{-}}$, and $\mathrm{Si}_{41}$-NCs. This work also investigates the effect of oxygen-related defects in such configurations. ${ }^{22}$ Ramos et al. conclude that defect-related phenomena do not explain the redshifts in the absorption spectra found by Wolkin et al. $^{3}$ but oxide or hydroxyl shells do, at least to some extent.

Despite the extensive research into Si-NCs embedded in silicon oxide matrices, a thorough understanding of the underlying mechanisms for their optical properties is lacking even in the simplest cases. Our aim, therefore, is to obtain unique insight through systematic real-time time-dependent density-functional theory (TDDFT) photoabsorption calculations for small highly symmetric oxide-embedded or hydrogen-passivated Si-NCs. The TDDFT method is a stateof-the-art method for calculating absorption spectra of molecules and atomic clusters. Other methods that have been previously used to calculate optical properties of nanostructures include the GW-Bethe-Salpeter equation (BSE) approach based on the Green's function method ${ }^{23}$ and the quantum-Monte Carlo method. ${ }^{24}$

This paper is organized as follows. Section II introduces briefly the utilized methods for calculating the absorption spectra. The optimization of the geometrical configuration of the Si-NCs is reviewed in Sec. III. In Sec. IV the results of this work are presented and discussed and in Sec. V the conclusions of this work are drawn. 
TABLE I. Bond lengths (in ångströms)

\begin{tabular}{|c|c|c|c|c|c|}
\hline \multirow[b]{2}{*}{ Species } & \multicolumn{3}{|c|}{$\mathrm{Si}-\mathrm{Si}$} & \multirow[b]{2}{*}{$\mathrm{Si}-\mathrm{O}$} & \multirow[b]{2}{*}{$\mathrm{Si}-\mathrm{H}$} \\
\hline & Inner & & Outer & & \\
\hline \multicolumn{6}{|c|}{ Hydrogen passivated: } \\
\hline $\mathrm{Si}_{5} \mathrm{H}_{12}$ & 2.370 & & & & 1.524 \\
\hline $\mathrm{Si}_{17} \mathrm{H}_{36}$ & 2.407 & 2.367 & & & 1.51 \\
\hline $\mathrm{Si}_{29} \mathrm{H}_{36}$ & 2.380 & 2.377 & 2.368 & & $1.51-1.52$ \\
\hline $\mathrm{Si}_{35} \mathrm{H}_{36}$ & 2.373 & 2.371 & $2.36-2.37$ & & 1.52 \\
\hline $\mathrm{Si}_{41} \mathrm{H}_{60}$ & 2.420 & 2.384 & $2.361-2.363$ & & $1.51-1.52$ \\
\hline $\mathrm{Si}_{47} \mathrm{H}_{60}$ & 2.427 & 2.380 & $2.362-2.365$ & & $1.51-1.52$ \\
\hline \multicolumn{6}{|l|}{ One oxide shell: } \\
\hline $\mathrm{Si}_{5} @ \mathrm{Si}_{12} \mathrm{O}_{12} \mathrm{H}_{36}$ & 2.338 & & & $1.66-1.68$ & $1.50-1.51$ \\
\hline $\mathrm{Si}_{17} @ \mathrm{Si}_{24} \mathrm{O}_{36} \mathrm{H}_{60}$ & 2.609 & 2.389 & & $1.66-1.69$ & $1.50-1.51$ \\
\hline $\mathrm{Si}_{29} @ \mathrm{Si}_{30} \mathrm{O}_{36} \mathrm{H}_{84}$ & 2.400 & 2.425 & 2.417 & $1.66-1.69$ & $1.50-1.51$ \\
\hline $\mathrm{Si}_{35} @ \mathrm{Si}_{36} \mathrm{O}_{36} \mathrm{H}_{108}$ & 2.358 & 2.374 & 2.387 & $1.68-1.70$ & $1.50-1.51$ \\
\hline $\mathrm{Si}_{41} @ \mathrm{Si}_{42} \mathrm{O}_{60} \mathrm{H}_{108}$ & 2.521 & 2.470 & $2.375-2.399$ & $1.66-1.70$ & $1.50-1.51$ \\
\hline $\mathrm{Si}_{47} @ \mathrm{Si}_{48} \mathrm{O}_{72} \mathrm{H}_{108}$ & 2.521 & 2.430 & $2.403-2.407$ & $1-65-1.71$ & $1.49-1.51$ \\
\hline \multicolumn{6}{|l|}{ Two oxide shells: } \\
\hline $\mathrm{Si}_{5} @ \mathrm{Si}_{36} \mathrm{O}_{48} \mathrm{H}_{60}$ & 2.354 & & & $1.65-1.69$ & $1.50-1.51$ \\
\hline $\mathrm{Si}_{17} @ \mathrm{Si}_{66} \mathrm{O}_{96} \mathrm{H}_{108}$ & 2.713 & 2.390 & & $1.64-1.70$ & $1.50-1.51$ \\
\hline
\end{tabular}

\section{METHOD OF CALCULATION}

The real-time TDDFT implementation is based on the scheme by Yabana and Bertsch. ${ }^{25}$ All electron states are excited by applying an instantaneous kick of momentum $\kappa$ at time $t=0$. These states are then let to evolve in time according to the time-dependent Kohn-Sham equations and the dipole moment $p(t)$ of the system is recorded. When $\kappa$ is small enough, only the dipole-moment response is induced. The dipole-strength function is

$$
S(\omega)=\frac{2 \omega}{\pi} \operatorname{Im}[\alpha(\omega)]
$$

where the dynamical polarizability $\alpha$ is proportional to the dipole moment as

$$
\alpha(\omega)=\frac{1}{\kappa} \int e^{i \omega t}[p(t)-p(0)] d t .
$$

A more thorough description of the TDDFT methods can be found, e.g., in Ref. 26 and in references therein.

The geometries of the studied structures in their electronic ground state are optimized with the ground-state DFT program SIESTA. ${ }^{27,28}$ It is a code based on linear combinations of atomic orbitals using the standard self-consistent KohnSham method. We use double-zeta-plus-polarization basis sets. The TDDFT calculations are carried out by the OCTOPUS program, ${ }^{29}$ which is particularly designed for TDDFT calculations. It employs a real-space grid and thus uses no atomic-orbital basis. In the TDDFT calculations, the simulation volume extends to at least $8 \AA$ around every nucleus. The simulation volume is divided into a regular grid with a grid spacing of $0.23 \AA$. To verify that this grid spacing is adequate, convergence tests are carried out for the smallest studied oxygen-coated $\mathrm{Si}-\mathrm{NC} \mathrm{Si}_{5} @ \mathrm{Si}_{12} \mathrm{O}_{12} \mathrm{H}_{36}$ with grid spacings of $0.23 \AA, 0.20 \AA$, and $0.17 \AA$. They show a scatter of less than $0.1 \mathrm{eV}$ for the lowest-absorption energies, thus ensuring the validity of the chosen grid spacing. The wave functions are propagated with a time step of 0.002 [small NCs (Ref. 30)] or $0.0025 \hbar / \mathrm{eV}$ (large NCs) for a total propagation time of $50 \hbar / \mathrm{eV} \approx 33$ fs (small NCs) or $37.5 \hbar / \mathrm{eV} \approx 25$ fs (large $\mathrm{NCs}$ ). In all calculations, the Perdew-Burke-Ernzerhof density-gradient-corrected semilocal exchange-correlation functional ${ }^{31}$ is used. Normconserving pseudopotentials of the improved TroullierMartins type ${ }^{32}$ are used throughout the calculations.

\section{GEOMETRY OPTIMIZATION}

Following partly the computational works reviewed in Sec. I, we have chosen a representative series of highly symmetric Si-NCs to our study. We consider hydrogenpassivated $\mathrm{Si}-\mathrm{NCs}$ and $\mathrm{Si}-\mathrm{NCs}$ embedded in one neutral $\mathrm{SiO}_{2}$ shell. For the two smallest Si-NCs, calculations with two $\mathrm{SiO}_{2}$ shells also are performed. The outermost layer of a $\mathrm{Si} @ \mathrm{SiO}_{2}$ cluster is always hydrogen passivated in order to get rid of dangling bonds and to better mimic the effect of bulk silicon oxide. The full list of studied structures is presented in Table I with their calculated bond lengths. Some of the structures are illustrated in Fig. 1. The diameter of the studied cores ranges from about $0.5 \mathrm{~nm}$ for $\mathrm{Si}_{5}$ to about $1 \mathrm{~nm}$ for $\mathrm{Si}_{47}$. The calculated bond lengths are in accordance with those calculated in Ref. 20 for hydrogen-passivated and oxide-embedded $\mathrm{Si}_{5^{-}}, \mathrm{Si}_{17^{-}}$, and $\mathrm{Si}_{41^{-}}$-NCs. The overall trend of slightly shorter bond lengths of Ref. 20 is due to the use of the local-density approximation (LDA) in the exchange- 


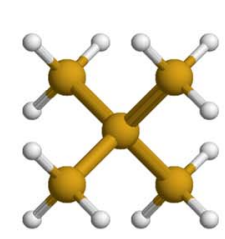

a)

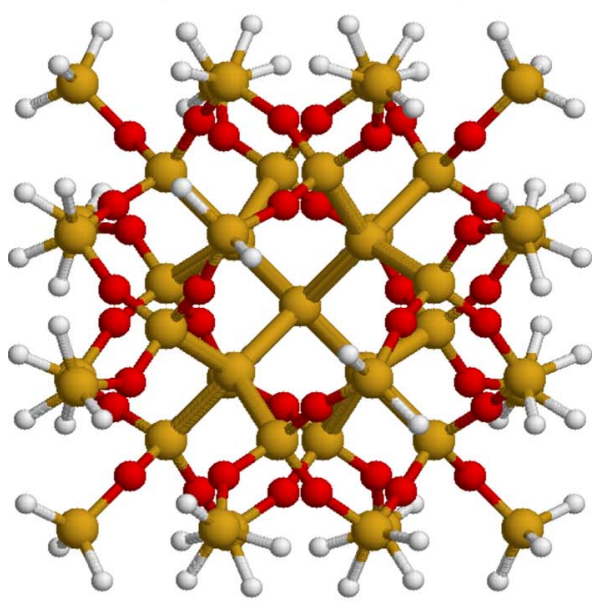

c)

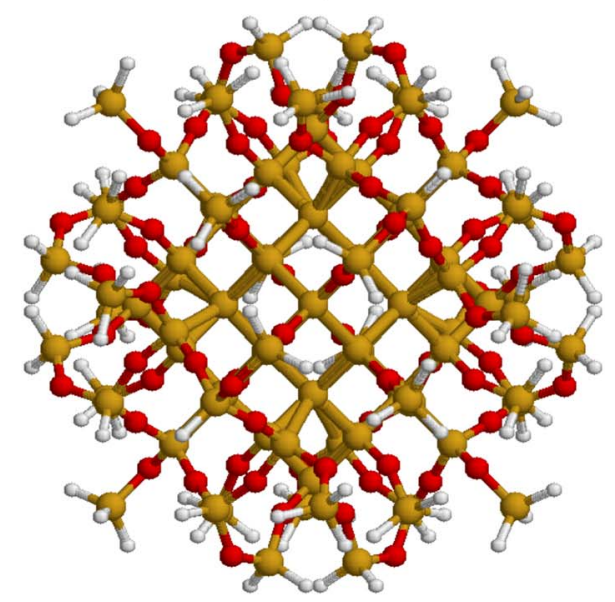

d)

FIG. 1. (Color online) Examples of structures of the studied Si-NCs are (a) $\mathrm{Si}_{5} \mathrm{H}_{12}$, (b) $\mathrm{Si}_{17} \mathrm{H}_{36}$, (c) $\mathrm{Si}_{17} @ \mathrm{Si}_{24} \mathrm{O}_{36} \mathrm{H}_{60}$, and (d) $\mathrm{Si}_{47} @ \mathrm{Si}_{48} \mathrm{O}_{72} \mathrm{H}_{108}$. The yellow spheres are $\mathrm{Si}$ atoms, the red spheres are $\mathrm{O}$ atoms, and the white spheres are $\mathrm{H}$ atoms. The plane of the paper is the plane spanned by two of the main axes of the NCs.

correlation potential, which is known to underestimate the bond lengths of the Si-NCs.

\section{RESULTS AND DISCUSSION}

The calculated absorption spectra of the studied systems are shown in Figs. 2 and 3. All the shown spectra consist of a gap region at the lowest energies, characteristic excitations in the energy range of about 3-6 eV, and a broad and rather nonspecific feature above the ionization energy ranging up to

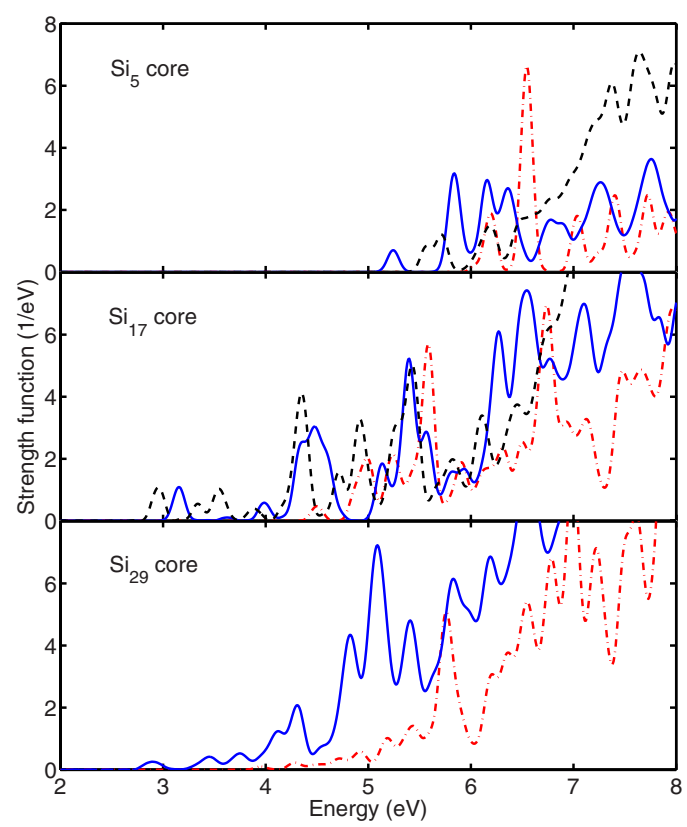

FIG. 2. (Color online) Calculated photoabsorption spectra of structures with $\mathrm{Si}_{5}, \mathrm{Si}_{17}$, and $\mathrm{Si}_{29}$ cores. The dash-dotted lines represent hydrogen-passivated $\mathrm{Si}-\mathrm{NCs}$, the solid lines represent $\mathrm{Si}-\mathrm{NCs}$ with one oxide shell, and the dashed lines represent $\mathrm{Si}-\mathrm{NCs}$ with two oxide shells.

several tens of eV. Clearly, the absorption spectra of the oxide-embedded Si-NCs differ significantly from those of the hydrogen-passivated ones. When the silicon-oxide shell covers the $\mathrm{Si}-\mathrm{NC}$, the minimum absorption energy decreases

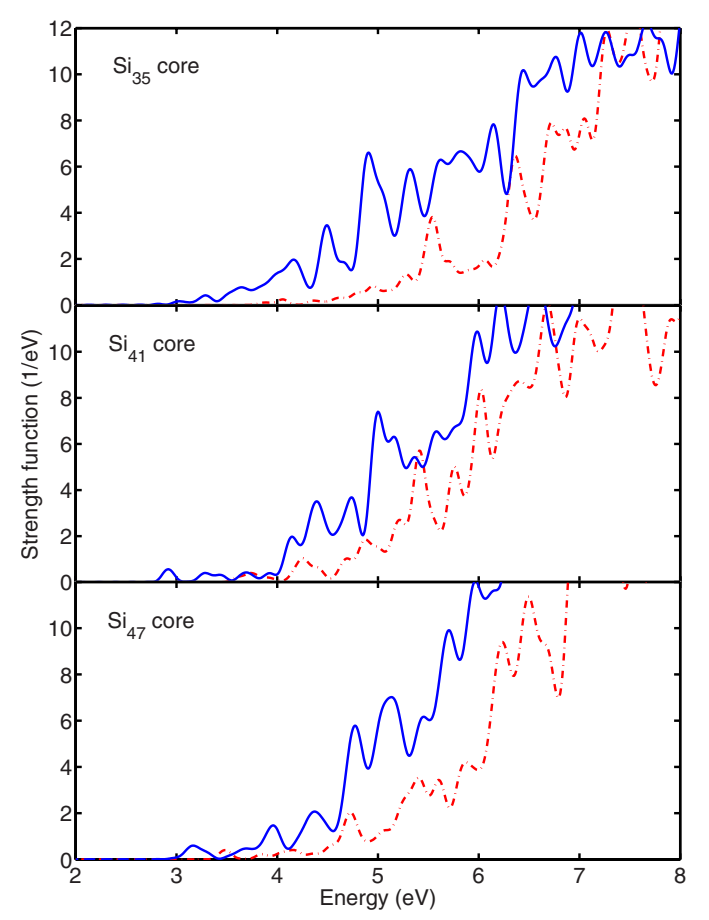

FIG. 3. (Color online) Calculated photoabsorption spectra of structures with $\mathrm{Si}_{35}, \mathrm{Si}_{41}$, and $\mathrm{Si}_{47}$ cores. The dash-dotted lines represents hydrogen-passivated $\mathrm{Si}-\mathrm{NCs}$ and the solid lines represent Si-NCs with one oxide shell. 


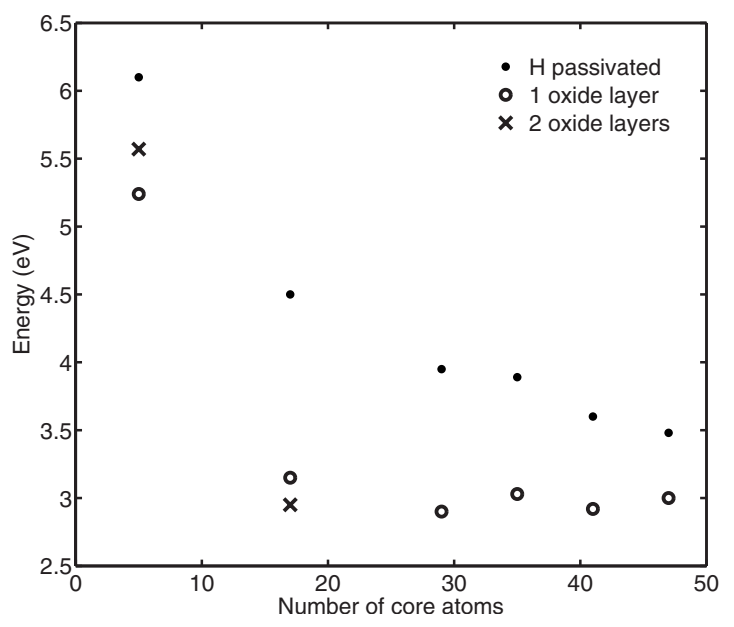

FIG. 4. Optical-gap energies of hydrogen-passivated Si-NCs (dots), $\mathrm{Si}-\mathrm{NCs}$ with one $\mathrm{SiO}_{2}$ layer (circles), and $\mathrm{Si}-\mathrm{NCs}$ with two $\mathrm{SiO}_{2}$ layers (crosses).

and the overall spectral pattern changes significantly compared to the hydrogen-passivated Si-NC.

The addition of a second oxide shell causes further variation in the absorption spectra in the two studied cases. This change is quite large in the case of the extremely small $\mathrm{Si}_{5}$-NC. However, for the $\mathrm{Si}_{17}-\mathrm{NC}$, the differences are already minor in the energy range of the main characterics at $2-6 \mathrm{eV}$. This shows that one oxide shell is enough to mimic the surrounding silicon oxide fairly reliably for the larger studied structures.

The optical gaps of the studied Si-NCs are presented in Fig. 4. This quantity is of great interest as it defines whether there is any optical activity in the interesting visible range. Although there has been discussion about how to define the optical gap in clusters and nanocrystals (see, e.g., Ref. 33), we have defined the optical gap simply as the energy at which the first photoabsorption peak raises to about a factor of ten above the numerical background noise.

We observe that for the hydrogen-passivated Si-NCs, the optical gap increases when the crystal size decreases. Our results agree well, within $0.5 \mathrm{eV}$, with the TDDFT results in Refs. 8 and 34-37 and are about $1 \mathrm{eV}$ lower than the optical gaps obtained with BSE (Ref. 38) and diffusion-Monte Carlo ${ }^{34}$ methods. For example, for $\mathrm{Si}_{17} \mathrm{H}_{36}$, our result of 4.5 $\mathrm{eV}$ is in accordance with the TDLDA result of $4.3 \mathrm{eV}$ by Benedict et al. ${ }^{34}$ whereas their diffusion-Monte Carlo result is $5.6 \mathrm{eV}$. For $\mathrm{Si}_{41} \mathrm{H}_{60}$, our result of $3.7 \mathrm{eV}$ is between the TDLDA result of $3.29 \mathrm{eV}$ and the time-dependent-HeydScuseria-Ernzerhof result of $4.02 \mathrm{eV}$ by Ramos et al. ${ }^{35}$ but is clearly lower than their quasiparticle-BSE result of $6.24 \mathrm{eV}$.

For the oxygen-embedded Si-NCs, we observe that the optical gap is constant, about $3 \mathrm{eV}$, as the size of the crystal decreases to 17 atoms. This result is surprising since it differs qualitatively from the behavior of the hydrogen-passivated Si-NCs. This trend is not clearly seen in the results of Ref. 20, where a few oxide-embedded Si-NCs are studied within the independent-particle approximation

The observed qualitative difference between hydrogenpassivated and oxide-embedded $\mathrm{Si}$-NCs means that the

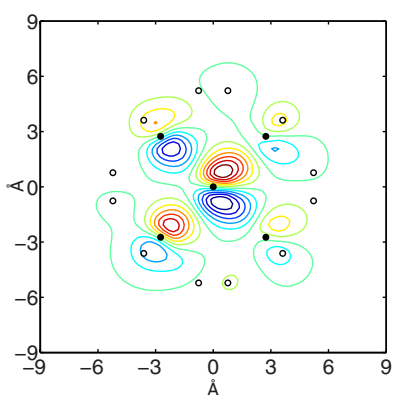

a)

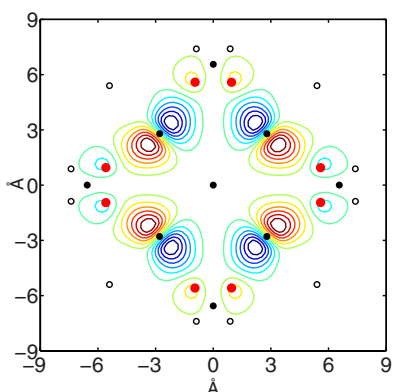

c)

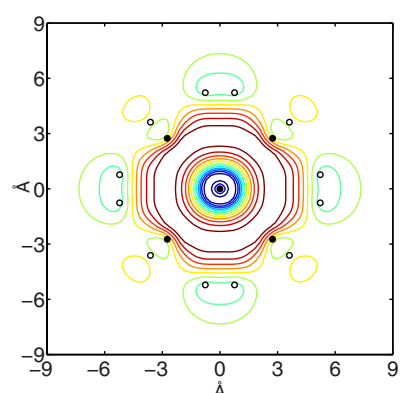

b)

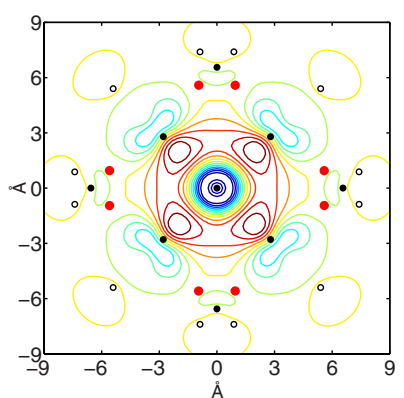

d)
FIG. 5. (Color online) Cuts of the HOMOs [(a) and (c)] and LUMOs [(b) and (d)] of $\mathrm{Si}_{29} \mathrm{H}_{36}$ [(a) and (b)] and $\mathrm{Si}_{29} @ \mathrm{Si}_{30} \mathrm{O}_{36} \mathrm{H}_{84}$ $[(\mathrm{c})$ and (d)] in the $x=0$ plane. The black dots, the large red dots, and the circles represent the silicon, oxygen, and hydrogen atoms in the vicinity of the plane, respectively.

mechanism producing the first optically allowed excitations is different in these two cases. To find out the origin of this difference, the electronic states are examined. A few HOMOs and LUMOs for the studied structures are examined in order to find out whether the oxidization has a systematic effect on them. Typical examples are shown in Fig. 5. We observe that for the hydrogen-passivated structures, the HOMOs are distributed all over the $\mathrm{Si}-\mathrm{NC}$, which is consistent with the results in Ref. 9. On the contrary, in the case of oxide-shell structures, the HOMOs are strongly localized in the vicinity of the Si-NC-oxide interface. No such difference is observed for the LUMOs. The first LUMO typically has a radially rather smooth shape for both hydrogen-passivated and oxideembedded structures. The difference in the localization of the HOMOs supports the idea that the effects at the silicon-oxide interface have an essential influence on the optical properties of Si-NCs. The shape of the HOMOs and LUMOs for the oxide-embedded Si-NCs agree well with those of the hydrogen-passivated and oxide-embedded $\mathrm{Si}_{17}-\mathrm{NCs}$ found by Ramos et al..$^{20}$ and the defect-imposed HOMOs and LUMOs of the oxide-embedded $\mathrm{Si}_{41}$-NC. ${ }^{22}$

In order to find out the specific transitions of the lowest optical-absorption energies, we calculated the transition matrices of the $\mathrm{Si}_{29}$ structures using the Casida method. ${ }^{39} \mathrm{We}$ find that the first excitation of the hydrogen-passivated $\mathrm{Si}_{29}$-NC is the HOMO-LUMO transition. The same is expected to hold for the other hydrogen-passivated Si-NCs because the optical gaps follow firmly the trend of the HOMOLUMO gaps. The situation is dramatically changed in the oxygen-embedded $\mathrm{Si}_{29}$-NC. As explained above, the HOMOs of the oxide-embedded Si-NCs are localized at the in- 
terface between the Si-NC and the oxide layer, whereas the LUMOs are localized at the center of the Si-NC. Thus, the transition between these states has a very small oscillator strength. Indeed, the first peak in the spectrum of $\mathrm{Si}_{29} @ \mathrm{Si}_{30} \mathrm{O}_{36} \mathrm{H}_{84}$ arises from transitions between the HOMO-(7-9) and the LUMO, the HOMO-(4-6) and the LUMO+(1-3), and the HOMO-(1-3) and the $\mathrm{LUMO}+(5-7)$. In fact, the HOMO is not participating in any transitions to low-lying unoccupied states. Also the single-electron spectrum near the HOMO-LUMO gap changes from the hydrogen-passivated to the oxideembedded Si-NCs. Namely, the energy intervals between the occupied and unoccupied eigenstates decrease. This, and the vanishing of transitions between certain eigenstates, results in a much more complex situation at lowest-excitation energies than that for hydrogen-passivated Si-NCs. The optical gap remains approximately constant when the Si-NC size changes.

We also note that in the example case of $\mathrm{Si}_{17}-\mathrm{NC}$, the thickness of the oxide cover does not have any major impact on the orbital characteristics. This supports our abovementioned conclusion that one surrounding oxide shell is enough to model reasonably the general case of a Si-NC embedded in silica.

\section{CONCLUSIONS}

We have calculated the photoabsorption spectra of a series of silicon nanocrystals embedded in silicon dioxide. Compared to hydrogen-passivated free-standing Si nanocrystals, the absorption spectra are shifted to lower energies by $0.5-$ $1.3 \mathrm{eV}$ depending on the cluster considered. They reach the visible range when the nanocrystal size is larger than about a dozen of atoms. However, the minimum absorption energy is constant, about $3 \mathrm{eV}$, as the size of the core is further increased. This trend is different from that of hydrogenpassivated structures for which the absorption edge decreases further as the size of the nanocrysal increases. The different trends reflect the increased complexity of low-energy excitations in oxide-embedded Si-NCs. It is due to the localization of HOMOs and a few other eigenstates below it at the nanocrystal-oxide interface.

\section{ACKNOWLEDGMENTS}

This research is supported by the Academy of Finland through the Centers of Excellence Program (2006-2011). CSC, the Finnish IT Center for Science, is acknowledged for providing computer resources through the Project No. tkk2035.
*Corresponding author; lak@fyslab.hut.fi

${ }^{1}$ Silicon Nanophotonics, 1st ed., edited by L. Khriachtchev (World Scientific Publishing, Singapore, 2008).

${ }^{2}$ L. T. Canham, Appl. Phys. Lett. 57, 1046 (1990).

${ }^{3}$ M. V. Wolkin, J. Jorne, P. M. Fauchet, G. Allan, and C. Delerue, Phys. Rev. Lett. 82, 197 (1999).

${ }^{4}$ L. Khriachtchev, M. Räsänen, S. Novikov, and J. Sinkkonen, Appl. Phys. Lett. 79, 1249 (2001).

${ }^{5}$ L. Dal Negro, M. Cazzanelli, L. Pavesi, S. Ossicini, D. Pacifici, G. Franzò, F. Priolo, and F. Iacona, Appl. Phys. Lett. 82, 4636 (2003).

${ }^{6}$ J. Ruan, P. M. Fauchet, L. Dal Negro, M. Cazzanelli, and L. Pavesi, Appl. Phys. Lett. 83, 5479 (2003).

${ }^{7}$ M. Cazzanelli, D. Navarro-Urriós, F. Riboli, N. Daldosso, L. Pavesi, J. Heitmann, L. X. Yi, R. Scholz, M. Zacharias, and U. Gösele, J. Appl. Phys. 96, 3164 (2004).

${ }^{8}$ I. Vasiliev, S. Öğüt, and J. R. Chelikowsky, Phys. Rev. Lett. 86, 1813 (2001).

${ }^{9}$ X. Wang, R. Q. Zhang, T. A. Niehaus, Th. Frauenheim, and S. T. Lee, J. Phys. Chem. C 111, 12588 (2007).

${ }^{10}$ A. Puzder, A. J. Williamson, F. A. Reboredo, and G. Galli, Phys. Rev. Lett. 91, 157405 (2003).

${ }^{11}$ A. Puzder, A. J. Williamson, J. C. Grossman, and G. Galli, Phys. Rev. Lett. 88, 097401 (2002).

${ }^{12}$ I. Vasiliev, J. R. Chelikowsky, and R. M. Martin, Phys. Rev. B 65, 121302(R) (2002).

${ }^{13}$ M. Luppi and S. Ossicini, Phys. Status Solidi A 197, 251 (2003).

${ }^{14}$ M. Luppi and S. Ossicini, Phys. Rev. B 71, 035340 (2005).

${ }^{15}$ M. Gatti and G. Onida, Phys. Rev. B 72, 045442 (2005).

${ }^{16}$ R. J. Eyre, J. P. Goss, and P. R. Briddon, Phys. Rev. B 76, 245325 (2007).
${ }^{17}$ L. Pavesi, L. Dal Negro, C. Mazzoleni, G. Franzò, and F. Priolo, Nature (London) 408, 440 (2000).

${ }^{18}$ S. Godefroo, M. Hayne, M. Jivanescu, A. Stesmans, M. Zacharias, O. I. Lebedev, G. Van Tendeloo, and V. V. Moshchalkov, Nat. Nanotechnol. 3, 174 (2008).

${ }^{19}$ N. Daldosso, M. Luppi, S. Ossicini, E. Degoli, R. Magri, G. Dalba, P. Fornasini, R. Grisenti, F. Rocca, L. Pavesi, S. Boninelli, F. Priolo, C. Spinella, and F. Iacona, Phys. Rev. B 68, 085327 (2003).

${ }^{20}$ L. E. Ramos, J. Furthmüller, and F. Bechstedt, Phys. Rev. B 70, 033311 (2004).

${ }^{21}$ L. E. Ramos, J. Furthmüller, and F. Bechstedt, Appl. Phys. Lett. 87, 143113 (2005).

${ }^{22}$ L. E. Ramos, J. Furthmüller, and F. Bechstedt, Phys. Rev. B 71, 035328 (2005).

${ }^{23}$ G. Onida, L. Reining, and A. Rubio, Rev. Mod. Phys. 74, 601 (2002).

${ }^{24}$ W. M. C. Foulkes, L. Mitas, R. J. Needs, and G. Rajagopal, Rev. Mod. Phys. 73, 33 (2001).

${ }^{25}$ K. Yabana and G. F. Bertsch, Phys. Rev. B 54, 4484 (1996).

${ }^{26}$ Time-Dependent Density Functional Theory, edited by M. A. L. Marques, C. A. Ullrich, F. Nogueira, A. Rubio, K. Burke, and E. K. U. Gross (Springer-Verlag, Berlin, 2006).

${ }^{27}$ P. Ordejón, E. Artacho, and J. M. Soler, Phys. Rev. B 53, R10441 (1996).

${ }^{28}$ J. M. Soler, E. Artacho, J. D. Gale, A. García, J. Junquera, P. Ordejón, and D. Sánchez-Portal, J. Phys.: Condens. Matter 14, 2745 (2002); http://www.uam.es/departamentos/ciencias/ fismateriac/siesta/

${ }^{29}$ A. Castro, H. Appel, M. Oliveira, C. A. Rozzi, X. Andrade, F. Lorenzen, M. A. L. Marques, E. K. U. Gross, and A. Rubio, 
Phys. Status Solidi B 243, 2465 (2006); http://www.tddft.org/ programs/octopus/

${ }^{30}$ In the calculations, the parameters for "small NCs" are used for hydrogen-passivated $\mathrm{Si}-\mathrm{NCs}$ with up to 35 atoms, oxygenembedded $\mathrm{Si}_{5}$-NCs, and for $\mathrm{Si}_{17} @ \mathrm{Si}_{24} \mathrm{O}_{36} \mathrm{H}_{60}$.

${ }^{31}$ J. P. Perdew, K. Burke, and M. Ernzerhof, Phys. Rev. Lett. 77, 3865 (1996).

${ }^{32}$ N. Troullier and J. L. Martins, Phys. Rev. B 43, 1993 (1991).

${ }^{33}$ I. Vasiliev, S. Öğüt, and J. R. Chelikowsky, Phys. Rev. B 65, 115416 (2002).

${ }^{34}$ L. X. Benedict, A. Puzder, A. J. Williamson, J. C. Grossman, G.
Galli, J. E. Klepeis, J.-Y. Raty, and O. Pankratov, Phys. Rev. B 68, 085310 (2003).

${ }^{35}$ L. E. Ramos, J. Paier, G. Kresse, and F. Bechstedt, Phys. Rev. B 78, 195423 (2008).

${ }^{36}$ L. Mitas, J. Therrien, R. Twesten, G. Belomoin, and M. H. Nayfeh, Appl. Phys. Lett. 78, 1918 (2001).

${ }^{37}$ D. Sundholm, Nano Lett. 3, 847 (2003).

${ }^{38}$ M. Rohlfing and S. G. Louie, Phys. Rev. Lett. 80, 3320 (1998).

${ }^{39}$ M. E. Casida, in Recent Developments and Application of Modern Density Functional Theory, edited by J. M. Seminario (Elsevier, Amsterdam, 1996), pp. 391-439. 